\title{
Oblique propagation of solitary electrostatic waves in magnetized plasmas with cold ions and nonthermal electrons
}

\author{
Frank Verheest ${ }^{1,2, a)}$ and Manfred A. Hellberg ${ }^{2, b)}$ \\ ${ }^{1}$ Sterrenkundig Observatorium, Universiteit Gent, Krijgslaan 281, B-9000 Gent, Belgium \\ ${ }^{2}$ School of Chemistry and Physics, University of KwaZulu-Natal, Durban 4000, South Africa
}

(Received 18 November 2016; accepted 26 January 2017; published online 16 February 2017)

Oblique propagation of large amplitude electrostatic waves and solitary structures is investigated in magnetized plasmas, comprising cold fluid ions and Cairns nonthermally distributed electrons, by using a Sagdeev pseudopotential formalism. To perform the analysis, quasineutrality is assumed, so that in normalized variables the electrostatic potential and the occurrence of solitary structures are governed by three parameters: the Mach number $M$, the typical Cairns parameter $\beta$, and the angle $\vartheta$ between the directions of propagation and the static magnetic field. Below a critical $\beta$, only positive compressive solitons are possible, and their amplitudes increase with increasing $\beta, M$, and $\vartheta$. Above the critical $\beta$, there is coexistence between negative rarefactive and positive compressive solitons, and the range of negative solitons, at increasing $M$, ends upon encountering a double layer or a singularity. The double layer amplitudes (in absolute value) increase with $\beta$ but are independent of $\vartheta$. Roots of the Sagdeev pseudopotential beyond the double layer are not accessible from the undisturbed conditions, because of an intervening singularity where the pseudopotential becomes infinite. Recent claims of finding supersolitons beyond a double layer appear to be based on a misinterpretation of the nature of the singularity. Published by AIP Publishing.

[http://dx.doi.org/10.1063/1.4976126]

\section{INTRODUCTION}

Oblique propagation of electrostatic waves and solitary structures in magnetized plasmas has, over the years, been investigated by several authors. ${ }^{1-14}$ The discussion has been carried out from different viewpoints: linear waves or weakly nonlinear solitons described by the reductive perturbation theory, or, less commonly, larger amplitude solitary structures treated through a Sagdeev pseudopotential approach. ${ }^{15}$ It is the latter method that interests us here even though some authors also mention linear dispersion properties and/or cite weakly nonlinear results (but derived as a limiting case from the fully nonlinear approach).

In order to get through the analysis, three assumptions are needed: only one fluid inertial species directly feels the influence of the static magnetic field, the inertia of the other species is neglected and their densities are obtained from a typical distribution, and one imposes quasineutrality. ${ }^{8}$ The latter means that in the algebra the overall charge density is mathematically zero, not only in equilibrium but also in the wave or nonlinear structure, yet there can be a wave electrostatic field. This assumption has to be critically evaluated at the end of the theoretical and numerical analysis, to get an idea of its applicability and limitations in this range of problems.

Moreover, because of the quasineutrality assumption, the nonlinear electrostatic modes discussed here cannot be considered of the acoustic type, contrary to the labels used sometimes, because one has essentially "lost" the Poisson equation. The latter is a cornerstone of investigations of nonlinear ion-

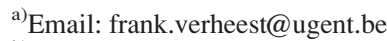

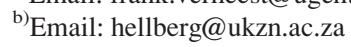

or electron-acoustic modes in unmagnetized plasmas or in magnetized plasmas when the propagation direction is parallel to the static field.

The present treatment thus implies that the transition from oblique to parallel propagation makes no sense. We therefore distinguish two physical directions, corresponding to those of the wave propagation and the static magnetic field. It is clear that one can orient the reference frame so that these two directions define the $\{x, z\}$ plane, with two possible choices for one of the axes, giving then an angle $\vartheta$ between the two physical directions. Proper orientation of the reference frame can ensure that $0<\vartheta<90^{\circ}$; however, $\vartheta$ cannot approach zero, implying that $\sin \vartheta \neq 0$. Below, we will argue that $\vartheta=90^{\circ}$ (and hence $\cos \vartheta=0$ ) too does not yield solutions.

Most of the papers studying oblique propagation of electrostatic modes in magnetized plasmas take the static field along one axis, thus leaving $x$ and $z$ as independent variables, in addition to time $t$. Later, a comoving coordinate is introduced to carry the analysis through, reducing the description to one effective coordinate. ${ }^{1-3,5,7,9-14}$

Another choice, one that we tend to prefer because it is simpler to work with, is that one axis is chosen along the direction of propagation and immediately in a comoving reference frame. ${ }^{4,6,8}$ Working in the frame where the structure is stationary gives rise to straightforward integrations with respect to $x$, as $t$ is eliminated from the very beginning. Obviously, with some adaptations of the boundary conditions, both approaches lead ultimately to the same result, which can be verified. All the papers surveyed rely on the Sagdeev pseudopotential method ${ }^{15}$ for the theoretical analysis and numerical evaluation. 
As to the plasma models discussed, the fluid constituent is usually positive ions, which are treated as strictly cold ${ }^{1,2,4-9,12,13}$ or warm, $3,10,14$ with various polytropic pressure-density relations, or else one cold electron component and an immobile background. ${ }^{11}$ The hot electrons are inertialess and have different distributions: in the early days, Boltzmann, ${ }^{1-5,7,8}$ more recently kappa, ${ }^{9,11,13}$ Tsallis, $^{12}$ or Cairns. ${ }^{14}$ We quote some generic references for these distributions. ${ }^{16-24}$ McKenzie $^{6}$ uses a general polytropic model for the hot electrons, whereas Shahmansouri and Alinejad ${ }^{13}$ consider a two-temperature kappa model for the electrons and Akbari-Moghanjoughi ${ }^{10}$ covers different distributions.

The motivation for the present work is the very recent paper by Rufai et al., ${ }^{14}$ who investigated a plasma model with warm ions (with inertia and an adiabatic pressuredensity thermodynamic relation) and nonthermal Cairns electrons. $^{24}$ Their paper is interesting in that both negative and positive solitary structures are obtained and even negative double layer and supersolitons.

That positive and negative solutions can coexist in the same plasma configuration has long been well established for acoustic type modes in unmagnetized plasmas (or equivalently, for parallel propagation in magnetized plasmas), but these treatments all rely on the full Poisson equation. ${ }^{25-27}$ Later, a distinction was made between KdV-like and nonKdV-like acoustic solitons. ${ }^{28-31}$

The KdV-like solitons share the typical property of $\mathrm{KdV}$ solitons, obtained through a reductive perturbation treatment, that as their velocity approaches the global acoustic speed from above, the amplitude decreases to zero. However, their amplitudes can grow large, as the weakamplitude constraint of the reductive perturbation method need not be heeded. To the contrary, nonKdV-like solitons can occur already at the global acoustic speed, with a nonzero amplitude, which increases as the soliton velocity becomes larger. Such solitons cannot be recovered from reductive perturbation analyses.

In acoustic soliton theory, double layers often act as a limit of a range of solitons, as their velocities and amplitudes increase. More rare is the occurrence of a soliton range beyond the double layer, which translates in a continuous increase of the velocity but a discontinuous jump in the amplitude. Superficially, electrostatic potential profiles in the first part of this range look like those of traditional solitons. However, their electric field profiles are markedly different, having additional extrema on the wings of the standard bipolar structure.

Pseudopotentials yielding solitons of this type were found earlier, ${ }^{29,30,32}$ but the associated electric field structures not investigated and thus not recognized, until Dubinov and Kolotkov drew attention to this characteristic and coined the name "super solitary wave" or supersoliton. ${ }^{33,34}$ Quite a number of papers then followed, in order to test a number of plasma compositions and parameter ranges that supported supersolitons. $^{35-42}$

We also investigated the possible occurrence of acoustic supersolitons in a plasma with only two constituents, fluid positive ions and hot electrons, ${ }^{40}$ for various electron distributions. The conclusion was that more plasma components than two were needed to facilitate the existence of acoustic supersolitons, in a model with sufficient adjustable parameters after a proper normalization.

Therefore, the claim that Rufai et al. ${ }^{14}$ observed supersolitons in a two-component plasma came as a surprise. Of course, their model includes the obliquity of the propagation with respect to a static magnetic field, and one might think that the resulting, more complex, pseudopotential could yield the multi-well shape required for supersolitons. However, the analysis is now in a different context: quasineutrality has replaced the use of Poisson's equation, and this precludes the smooth transition from oblique to parallel propagation.

The paper is structured as follows. After this Introduction, Sec. II gives the basic formalism, up to the derivation of the Sagdeev pseudopotential for a plasma model with cold positive ions in the presence of Cairns electrons. Experience has taught us that including fluid ion thermal properties is not paramount in determining qualitative changes in soliton characteristics but complicates the parametric investigation and delimitation of the existence domains. Hence, our choice is a cold fluid ion constituent, a simplification of the model of Rufai et al., ${ }^{14}$ that does not affect the fundamental physics. In Sec. III, some analytical properties of the Sagdeev pseudopotential are given, which will help in Sec. IV with the numerical evaluation of the soliton properties, their existence domains, and variation with compositional parameters and profiles. In Sec. V, we discuss aspects of the quasineutrality assumption underpinning the formalism, while Sec. VI summarizes our findings.

\section{BASIC FORMALISM}

Let us start from a two-constituent plasma model with cold ion fluid equations of continuity and motion, in normalized variables

$$
\begin{gathered}
\frac{d}{d x}\left(n u_{x}\right)=0, \\
u_{x} \frac{d \mathbf{u}}{d x}=\mathbf{E}+\mathbf{u} \times \mathbf{B},
\end{gathered}
$$

written in a frame comoving with the nonlinear electrostatic structure propagating along the $x$ axis. Here, $n$ and $\mathbf{u}$ are the ion density and fluid velocity and $\mathbf{E}$ and $\mathbf{B}$ are the electric field and magnetic induction, respectively. The original physical variables (not written) have been normalized as follows: density by the undisturbed ion and electron density (equal due to charge neutrality in equilibrium), velocities by $c_{i a}=\sqrt{T_{e} / m_{i}}$ in terms of the electron kinetic temperature and the ion mass, time by the inverse gyrofrequency $\Omega_{i}=e B_{0} / m_{i}$ involving the strength of the static induction, length by $c_{i a} / \Omega_{i}$, and the electrostatic potential $\phi$ by $T_{e} / e$. The normalizing speed, $c_{i a}$, is, of course, the ion-acoustic speed for a two-species plasma with cold ions and Boltzmann electrons $(\beta=0)$, not Cairns electrons.

Because we are investigating electrostatic modes, there are no wave magnetic effects and there only remains a static induction, oriented for oblique propagation as $\mathbf{B}_{0}=\mathbf{e}_{x} \cos \vartheta$ $+\mathbf{e}_{z} \sin \vartheta \equiv \mathbf{e}_{B}$, with $\vartheta$ the angle between the directions of 
wave propagation and static field. The undisturbed ions move by at a normalized velocity $M=V / c_{i a}$ along the $x$ axis, where $V$ is the solitary structure speed (as measured in an inertial frame), assumed to exist but not yet determined.

In the comoving frame, the electric field has two components. The undisturbed form of the equations of motion (2) indicates that we need

$$
\mathbf{E}_{0}+M \mathbf{e}_{x} \times \mathbf{e}_{B}=0,
$$

so that there is a constant electric field ${ }^{6} E_{0 y}=M \sin \vartheta$. On the other hand, the wave electric field is assumed to be curlfree and hence occurs as a potential gradient, which only has an $x$ component, $-d \varphi / d x$. This has been used even though we invoke the quasineutrality assumption. Disregarding Poisson's equation, however, will impose limitations on the obliquity of the propagation, ${ }^{8}$ to be discussed below.

The electrons obey a nonthermal Cairns distribution ${ }^{24}$

$$
n=\left(1-\beta \varphi+\beta \varphi^{2}\right) \exp [\varphi],
$$

and because of quasineutrality, we use the same symbol $n$ for both the electron and the ion density. By describing the electrons as inertialess, obeying an extension of the ubiquitous Boltzmann distribution, we implicitly neglect the magnetic part of the Lorentz force in the electron dynamics. However, a simple physical model ${ }^{43}$ suggests that ignoring electron inertia is justifiable if one avoids near-perpendicular propagation, while kinetic theory with inertial electrons ${ }^{44,45}$ yields linear low frequency ion-acoustic modes $\left(\omega<\Omega_{i}\right)$ that are analogous to those found for unmagnetized plasmas with inertialess electrons. We will accept that such arguments are also valid in the nonlinear regime and will return to this aspect in Sec. VI, when dealing with the limitations of the model.

The ion equation of continuity (1) can immediately be integrated to yield the conservation of (mass) flux

$$
n u_{x}=M .
$$

The boundary conditions for solitary structures are $n \rightarrow 1, u_{x} \rightarrow M$, and $\varphi \rightarrow 0$, far away from the nonlinear disturbance. The other velocity components need $u_{y} \rightarrow 0$ and $u_{z} \rightarrow 0$. Here, the generic outline of an earlier paper $^{8}$ is followed, adapted to our two-component model.

We write the components of (2) as

$$
\begin{gathered}
u_{x} \frac{d u_{x}}{d x}+\frac{d \varphi}{d x}=u_{y} \sin \vartheta \\
u_{x} \frac{d u_{y}}{d x}=\left(M-u_{x}\right) \sin \vartheta+u_{z} \cos \vartheta \\
u_{x} \frac{d u_{z}}{d x}=-u_{y} \cos \vartheta .
\end{gathered}
$$

Before going on, we note that for parallel propagation $(\vartheta=0)$, (6) becomes decoupled from (7) and (8). Multiplying (7) by $u_{y}$ and (8) by $u_{z}$ and adding the resultant equations yields

$$
\frac{d}{d x}\left(u_{y}^{2}+u_{z}^{2}\right)=0
$$

In view of the boundary conditions, integration leads to $u_{y}=u_{z}=0$. Of (6) only the left hand side remains, and it can be expressed with the help of (5) as

$$
F \frac{d \varphi}{d x}=0,
$$

where

$$
F \equiv 1-\frac{M^{2}}{n^{3}} \frac{d n}{d \varphi}
$$

The expression for $F$ will also be used below, and we retain $n$ as shorthand for the function of $\varphi$ given by (4).

In the case of parallel propagation, $F$, a function of $\varphi$, is zero. Thus, only a constant root is possible, not a function of $x$, and in view of the boundary conditions, one obtains $\varphi=0$. This should come as no surprise, because the quasineutrality condition voids the acoustic character of the electrostatic mode.

Running ahead of ourselves, we would like to point out that we have not been able to generate nonlinear structures for perpendicular propagation, in line with earlier remarks in the literature. ${ }^{13}$ Most other authors do not explicitly address this aspect. ${ }^{8}$

Elimination of $u_{y}$ between (6) and (8) gives, after multiplication by $n$, that

$$
\frac{d u_{x}}{d x} \cos \vartheta+\frac{d u_{z}}{d x} \sin \vartheta+n \frac{\cos \vartheta}{M} \frac{d \varphi}{d x}=0 .
$$

This can be integrated to

$$
\left(u_{x}-M\right) \cos \vartheta+u_{z} \sin \vartheta+\frac{\cos \vartheta}{M} \int_{0}^{\varphi} n d \tilde{\varphi}=0,
$$

where $\tilde{\varphi}$ is a dummy integration variable. We eliminate $u_{z}$ between (7) and (13) to obtain

$$
\frac{d u_{y}}{d x} \sin \vartheta+G=0
$$

in terms of

$$
G \equiv 1-n+n \frac{\cos ^{2} \vartheta}{M^{2}} \int_{0}^{\varphi} n d \tilde{\varphi} .
$$

Elimination of $u_{y}$ between (6) and (14) yields

$$
\frac{d}{d x}\left(F \frac{d \varphi}{d x}\right)+G=0 .
$$

After multiplying (16) by $F d \varphi / d x$, the resulting expression is readily integrated and leads to a Sagdeev-type energy integral

$$
\frac{1}{2}\left(\frac{d \varphi}{d x}\right)^{2}+S(\varphi)=0
$$

where the Sagdeev pseudopotential is

$$
S(\varphi)=\frac{1}{F(\varphi)^{2}} \int_{0}^{\varphi} F(\tilde{\varphi}) G(\tilde{\varphi}) d \tilde{\varphi}
$$


For further discussions, we use the abbreviation

$$
H=\int_{0}^{\varphi} F(\tilde{\varphi}) G(\tilde{\varphi}) d \tilde{\varphi} .
$$

From (18), it is clear that $S(\varphi)$ has a singularity for the roots of $F=0$, and these potential value(s), $\varphi_{\ell}$, will play an important role. Interestingly, as can be seen from the definition of $F$, the existence of such singularities is generic for any magnetized plasma model in which the inertialess electrons can be represented as $n(\varphi)$.

Writing $S(\varphi)$ as a function of $\varphi$ in full glory yields a very cumbersome expression, which is, moreover, physically not at all enlightening. In order to avoid typographical and other errors, also in the programming of the numerical evaluation, we prefer to base our reasoning on the compact expressions $n, F, G$, and $H$, given in (4), (11), (15), and (19), respectively. As an aside, if ion thermal effects are included, the expressions of $F$ and $G$, and hence also $H$, become more complicated, but this does not invalidate the generic structure of the resulting Sagdeev pseudopotential $S .{ }^{8}$

\section{ANALYSIS}

Denoting derivatives with respect to $\varphi$ by dashes, and for the sake of brevity not writing the dependence on the variable $\varphi$ and parameters explicitly, we find that

$$
\begin{gathered}
S^{\prime}=\frac{G-2 S F^{\prime}}{F}, \\
S^{\prime \prime}=\frac{F G^{\prime}-G F^{\prime}-2 S^{\prime} F F^{\prime}-2 S F F^{\prime \prime}+2 S F^{\prime 2}}{F^{2}} .
\end{gathered}
$$

As $S(0)=0$ and $G(0)=0$, it follows that the undisturbed values yield $S^{\prime}(0)=0$ and

$$
S^{\prime \prime}(0)=\frac{G^{\prime}(0)}{F(0)}=\frac{\cos ^{2} \vartheta-(1-\beta) M^{2}}{M^{2}\left[1-(1-\beta) M^{2}\right]} \leq 0,
$$

where the inequality applies for solitary waves. We assume that $M$ is such that $F(0) \neq 0$. In order to have solitary modes, one needs an unstable maximum of $S(\varphi)$ in the undisturbed conditions at $\varphi=0$, so that from (22) the limits on $M$ are

$$
M_{c}=\frac{\cos \vartheta}{\sqrt{1-\beta}} \leq M<\frac{1}{\sqrt{1-\beta}}=M_{x} .
$$

The lower limit, $M_{c}$, represents the true acoustic speed in this plasma. ${ }^{14,45}$ It includes the $\beta$-dependence from the Cairns electron distribution function and a factor $\cos \vartheta$ typical of oblique propagation in a magnetic field. ${ }^{9,45}$ The upper limit, $M_{x}$, is the true acoustic speed in an equivalent unmagnetized Cairns plasma. ${ }^{9}$

All this allows us to compute

$$
\left.S^{\prime \prime \prime}(0)\right|_{M=M_{c}}=\left.\frac{G^{\prime \prime}(0)}{F(0)}\right|_{M=M_{c}}=\frac{3(1-\beta)^{2}-1}{\sin ^{2} \vartheta} .
$$

We know that the sign of $\left.S^{\prime \prime \prime}(0)\right|_{M=M_{c}}$ gives the polarity of the KdV-like modes, positive $(\varphi>0)$ or negative $(\varphi<0) .{ }^{28,29,46-49}$ The numerator of $\left.S^{\prime \prime \prime}(0)\right|_{M=M_{c}}$ vanishes for $\beta_{c}=1-1 / \sqrt{3} \simeq 0.4227$. For $\beta<\beta_{c}$, one has positive polarity $\mathrm{KdV}$-like solitons, for $\beta>\beta_{c}$ the $\mathrm{KdV}$-like solitons have negative polarities. There might in the latter case also be positive nonKdV-like solitons, but that has to be determined in detail below. The critical $\beta_{c}$ is typical for the Cairns distribution and is also encountered in many other applications, mostly investigating nonlinear acoustic modes in unmagnetized plasmas.

Further remarks can be made to guide the analysis and numerical evaluation. It is clear that $S$ has a singularity for roots of $F$, and from (11) these are given by

$$
M^{2}=M_{\ell}^{2} \equiv n^{3}\left(\frac{d n}{d \varphi}\right)^{-1},
$$

where from (4) $d n / d \varphi=\left(1-\beta+\beta \varphi+\beta \varphi^{2}\right) \exp [\varphi]$. Again, the inclusion of ion thermal effects ${ }^{14}$ complicates $F$ and $M_{\ell}$, shifting the position of the singularity in $S$ but cannot do away with it completely.

Any roots of $S$ that may occur beyond this singularity cannot be reached from the undisturbed conditions $\varphi=0$, and thus they have no physical relevance: the traditionally invoked pseudoparticle cannot roll past a singularity in the potential well!

From (4), one can prove that $d n / d \varphi>0$ for all $0 \leq \beta$ $<4 / 5$. However, this value exceeds the maximum admissible $\beta=4 / 7 \simeq 0.5714$ at which the underlying Cairns distribution in velocity phase space starts to develop wings, which might lead to beam instabilities, ${ }^{50}$ preventing the occurrence of sustainable nonlinear structures. Hence, $M_{\ell}$ exists for all admissible $\beta$ and the singularities cannot be avoided: they always limit the available $\varphi$ range.

As may be seen from (25), $M_{\ell}$ is a function of $\varphi$ and $\beta$ but independent of $\vartheta$. This statement has to be qualified: it holds if $M$ is a freely adjustable parameter (within limits). However, in cases where $M$ is fixed, such as when a Sagdeev pseudopotential has a double root solution, yielding a double layer, the corresponding $M_{d l}$ is determined from the full Sagdeev pseudopotential, i.e., the angle $\vartheta$, too, plays a role. Moreover, it is possible for more than one singularity to be present, and whether the singularity occurs on the negative or positive $\varphi$ side is in general not predictable.

Hence, we will also be looking for double roots having $\varphi \neq 0$. For given $\beta$ and $\vartheta$, one has to find simultaneous roots $\{\varphi, M\}$ of $S$ and of $S^{\prime}$. However, roots of $S$ are given by roots of $H$, and vice versa, and (20) indicates that $S=S^{\prime}=0$ also implies $G=0$. Because $S=S^{\prime}=0 \Longleftrightarrow G=H=0$, it is numerically simpler and more stable to solve $G=H=0$ when determining double root parameters, for which one finally has to plot $S$.

One notes that $G=0$ yields

$$
\frac{M^{2}}{\cos \vartheta^{2}}=\frac{n}{n-1} \int_{0}^{\varphi} n d \tilde{\varphi} .
$$

Remarkably, the right hand side only depends on $\beta$ and $\varphi$, so that values of $M$ for which double layers can occur are such that the ratio $M_{d l} / \cos \vartheta$ is fixed for specific pairs of $\{\beta, \varphi\}$, as will be illustrated below. 


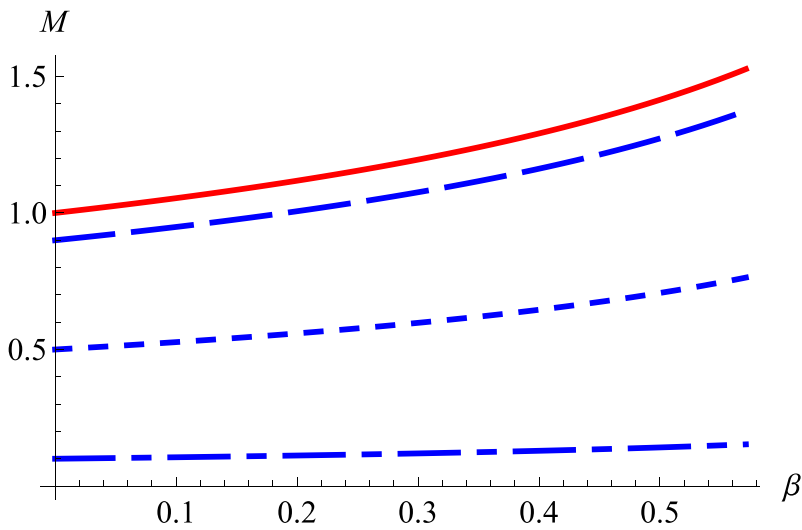

FIG. 1. Limits for $M$ as a function of $\beta$ from 0 to 0.571 , the value at which the Cairns distribution in velocity space starts to show a nonmonotonic behavior. The solid red curve is $M_{x}$, while $M_{c}$ is given by the blue dashed ( $\cos \vartheta=0.9)$, blue dotted $(\cos \vartheta=0.5)$, and blue dotted-dashed ( $\cos \vartheta=0.1)$ curves. The available $M$ range increases with $\vartheta$.

\section{NUMERICAL EVALUATION}

We start the numerical evaluation by plotting the minimum and maximum values of $M$ as a function of $\beta$ for three typical values of $\cos \vartheta$. Because of the property expressed by (26), it is more appropriate to present the graphs in terms of $\cos \vartheta$, rather than $\vartheta$ itself.

From Fig. 1, it is seen that one can find acceptable parameter values $\beta$ and $M$ over the ranges $0 \leq \beta \leq 0.5714$ and $M_{c} \leq M<M_{x}$, and this is, in principle, for all acceptable $\cos \vartheta \neq 0$. By acceptable, we mean that the model does not allow a transition to parallel propagation and also that there will be limitations for strong obliquity. Unfortunately, the latter cannot be quantified precisely, an aspect that will be discussed below.

The dependence of the double layer amplitudes and Mach numbers on $\cos \vartheta$ and $\beta$ is illustrated in Figs. 2 and 3, respectively. It is remarkable that, for fixed $\beta$, chosen in Fig. 2 as $\beta=0.5$, the double root amplitudes are independent of the propagation angle, expressed through $\cos \vartheta$. On the other hand, the Mach numbers, $M_{d l}$, vary linearly with $\cos \vartheta$, which is a reflection of the property expressed by (26) and another reason for presenting the graphs in terms of $\cos \vartheta$ rather than $\vartheta$ itself.

Turning now to the discussion of Fig. 3, when $\beta$ is varied over the domain where one expects double root solutions to occur, the absolute value of the amplitude increases with

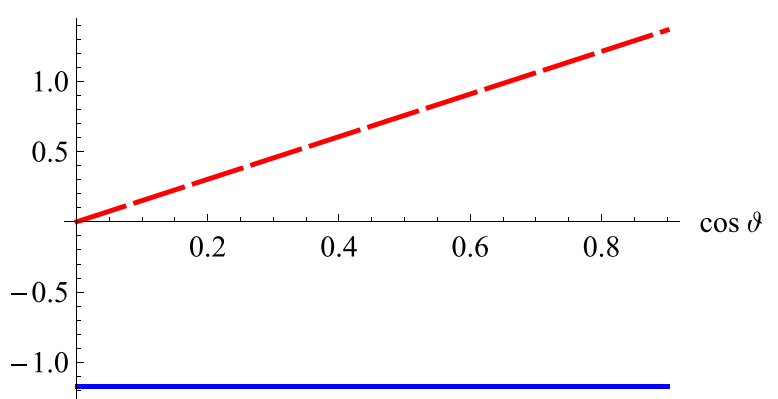

FIG. 2. Double layer amplitudes (solid blue, negative domain) and Mach numbers (dashed red, positive domain) as a function of $\cos \vartheta$ for a typical value of $\beta=0.5$.

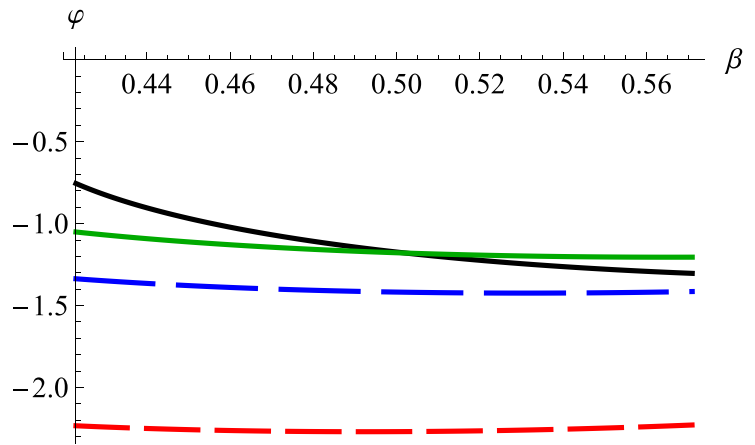

FIG. 3. Negative double layer amplitudes are represented by the solid black curve, and we have seen that these are valid for any obliquity at any given $\beta$. The other curves represent the amplitudes of the singularities (roots of $F=0$ ) for the parameter values $M_{d l}$ determining the double root solutions. These curves are coded as follows: solid green for $\cos \vartheta=0.9$, dashed blue for $\cos \vartheta=0.8$, and dotted black for $\cos \vartheta=0.5$.

$\beta$. As we are considering values of $\beta$ over which double layers may occur, we have chosen to plot the graphs over the range from $\beta_{c}=0.4227$ to $\beta=0.571$, where the underlying Cairns distribution in phase space begins to show a nonmonotonic character. ${ }^{50}$

An important remark here is that the green curve showing the values of $\varphi$ at which a singularity occurs for an obliquity $\cos \vartheta=0.9$ intersects the black curve for the double layer amplitudes (valid for all values of obliquity) at $\beta=0.502$, whereas the curves for more oblique propagation $(\cos \vartheta=0.8$ and 0.5 , and others not shown for graphical clarity) are completely below the double root curve. In the range $0.4227 \leq \beta<0.502$, the soliton amplitudes increase with $M$ from zero to the double layer amplitude, $\varphi_{d l}$, and the negative singularities, $\varphi_{\ell}$, lie beyond the double root. Hence, the double layer terminates a range of $\mathrm{KdV}$-like solitons, as has commonly been observed for ion-acoustic solitons in various plasma configurations. But for $0.502<\beta \leq 0.5714$, the reverse occurs: the singularity in $F$ is encountered first, and thus it limits the range of $\mathrm{KdV}$-like solitons.

As the obliquity is decreased, the point of intersection of the two curves is shifted to lower $\beta$ until it occurs at $\beta=$ 0.4227 for $\cos \vartheta=0.979$, i.e., $\vartheta=11.8^{\circ}$. Conversely, the intersection shifts to higher $\beta$ as the obliquity is increased, until it occurs at the maximum admissible value, $\beta=0.5714$ for $\cos \vartheta=0.852$, i.e., $\vartheta=31.6^{\circ}$. Thus, for obliquity below $11.8^{\circ}$ the singularity is always encountered before a double root can occur. Between $\vartheta=11.8^{\circ}$ and $31.6^{\circ}$, there is a crossover: for $\beta$ close to but larger than $\beta_{c}$, double roots are encountered before the singularity, whereas for $\beta$ near the upper limit of $4 / 7$, the singularity prevents the double root from occurring, and only negative solitons can exist. For all larger values of the obliquity $\left(\vartheta>31.6^{\circ}\right)$, the double root is always encountered before the singularity.

Moreover, the singularity exists for all angles of propagation, from near parallel to near perpendicular. As shown below, the pseudopotential curve representing the double layer (for $M=M_{d l}$ ) drops to $-\infty$ when $\varphi<\varphi_{d l}$. As Sagdeev pseudopotentials for $M>M_{d l}$ cannot cross the double layer curve,${ }^{47}$ these cannot have physically interesting negative roots. In particular, this rules out the possibility of 


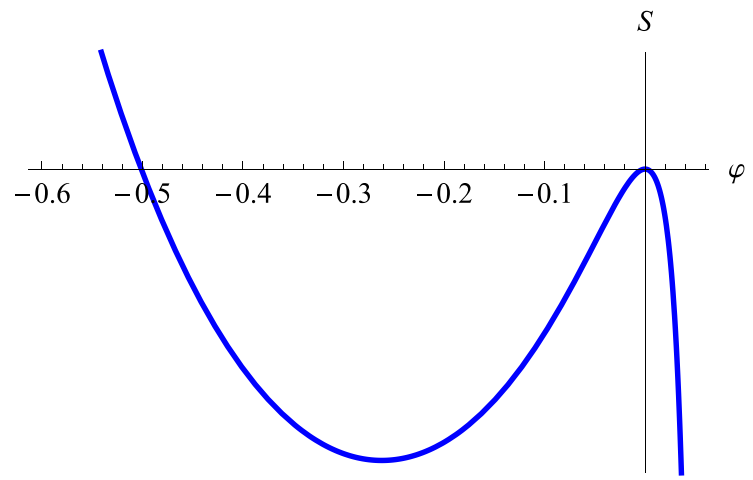

FIG. 4. Example of a Sagdeev pseudopotential for $\beta=0.55, \cos \vartheta=0.9$ and $M=1.45$, having only a negative $\mathrm{KdV}$-like soliton and no double layer.

having supersolitons, contrary to what has been claimed by Rufai et al. ${ }^{14}$ Although their model includes adiabatic pressure effects for the ions, these will modify only the quantitative details of the double roots and the singularities but cannot alter the qualitative picture that the singularity prevents physical roots from existing beyond the double root solutions.

This crossover between the relative values of $\varphi_{d l}$ and $\varphi_{\ell}$ for increasing $\beta$ does not occur for $\cos \vartheta=0.8$, as shown in Fig. 3. Therefore, a range of solitons will then always be limited by a double layer, with a singularity following at larger $|\varphi|$. The discussion below will use this value of $\vartheta$ to get the most interesting set of results.

However, before going into the details of those double layers, it is instructive to examine in Fig. 4 a Sagdeev pseudopotential for the combination of $\beta=0.55$ and $\cos \vartheta=0.9$, where on the negative side the soliton range is limited by a singularity. We choose $M=1.45$, lying between the limits $M_{c}=1.342$ and $M_{x}=1.491$, and find that $F=0$ has two singularities, at $\varphi=-1.284$ and at $\varphi=0.068$, with limits in $S$ at $+\infty$ and $-\infty$, respectively. For graphical clarity, the range in $\varphi$ has been curtailed, to avoid the two singularities on either side of the existence range from squashing the graph. Although there is a perfect negative KdV-like soliton, no nonKdV-like solution exists for the parameter values chosen.

Considering now $\cos \vartheta=0.8$, and starting with the lower $\beta<\beta_{c}=0.4227$ regime, one finds only positive $\mathrm{KdV}$ like solitons, two examples of which are illustrated in Fig. 5 for $\beta=0.25$. For those values, (4) indicates that $n>1$, and

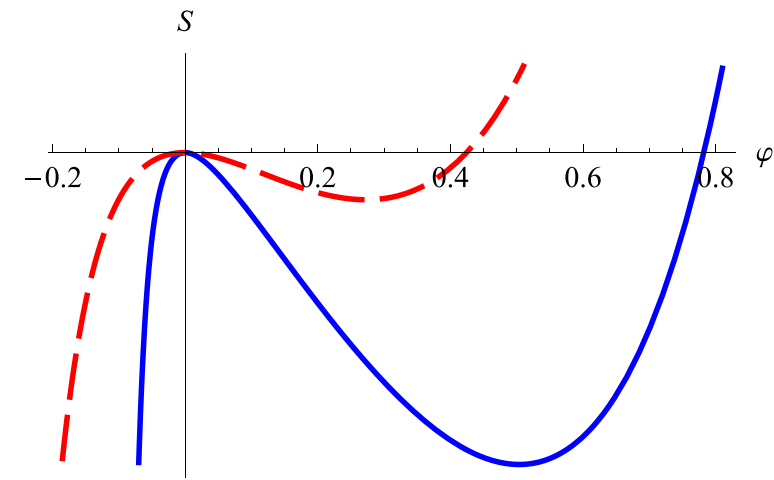

FIG. 5. Example of Sagdeev pseudopotentials for $\beta=0.25, \cos \vartheta=0.8$, and $M=1$ (red dashed curve) or $M=1.1$ (blue solid curve), having only a positive KdV-like soliton root. the modes are compressive. We can repeat this for other $\beta<\beta_{c}$, and other propagation angles, as long as $M$ lies between the corresponding $M_{c}$ and $M_{x}$, to find Sagdeev pseudopotentials, which are topologically equivalent to Fig. 5, yielding positive compressive solitons. In particular, this result, of course, reduces to the standard case of Boltzmanndistributed electrons, obtained by choosing $\beta=0$.

Turning next to the range $\beta>\beta_{c}$, we expect qualitative changes of the kind that are known from many studies of pure acoustic-type solitons and double layers in unmagnetized plasmas. A generic example of a Sagdeev pseudopotential with positive and negative solitons is shown in Fig. 6, in the domain where $\beta>\beta_{c}$ ensures the existence of double roots. To begin, for $\beta=0.5$ and $\cos \vartheta=0.8$, we note that $M_{c}=1.131$ is the minimum $M$ for which solitons might occur and also that $M_{d l}=1.214$ is the maximum.

The Sagdeev pseudopotential illustrated in Fig. 6 also shows a root at $\varphi=-1.520$, obviously at a larger amplitude than the double root solution. However, it is cut off from the Sagdeev potential well by the intervening singularity at $\varphi_{\ell}=-1.418$. Consequently, it cannot be accessed from the undisturbed conditions at $\varphi=0$. The curve in Fig. 6 extends beyond the singularity all the way for $\varphi \rightarrow-\infty$ but approaches zero from above: there are no further roots. On the positive potential side, one can prove that $\lim _{\varphi \rightarrow+\infty} S$ is $+\infty$.

Hence, values of $M$ larger than $M_{d l}=1.214$ might, erroneously, give the impression of Sagdeev pseudopotentials with a root beyond the singularity, but this is not accessible and without physical relevance. Thus, no solitons exist beyond the double layer, let alone supersolitons.

We suggest that the claim made by Rufai et al. ${ }^{14}$ that their model supports supersolitons beyond the double root solution is based on a misinterpretation of the nature of the singularity, presumably viewed as a very deep well (the bottom of which is not shown in their graphs). The main characteristics of their graphs correspond well to our Figs. 6 and 7.

As one would expect from our general expression (18), their Sagdeev potential $V(\psi, M)$ has singularities. These obviously occur at values of $\psi$ at which the first factor on the r.h.s. in their Eq. (18) vanishes. That factor is in essence our function $F$, with the added complexity of warm ions represented by $\sigma$. Using this expression, one may evaluate the singularity values $\psi_{\ell}$ for the "double layer" and "supersoliton"

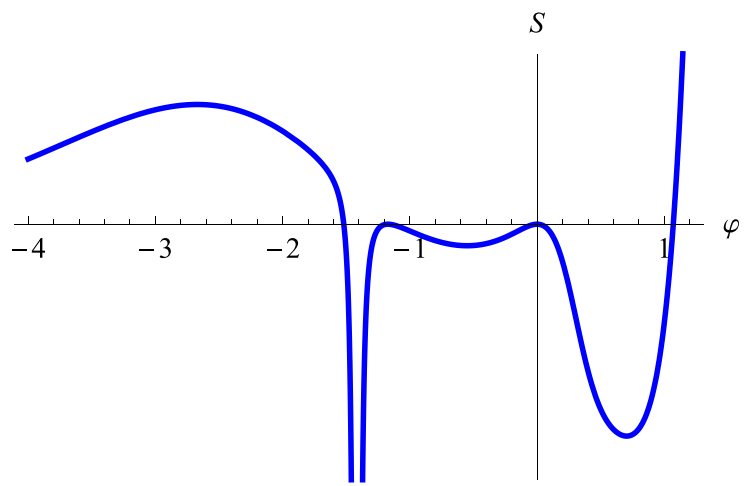

FIG. 6. Typical Sagdeev pseudopotential for $\beta=0.5, \cos \vartheta=0.8$ and $M=M_{d l}=1.214$. This has a nonKdV-like positive soliton at $\varphi=1.070$, a negative double root at $\varphi_{d l}=-1.173$, and a singularity at $\varphi_{\ell}=-1.418$. 


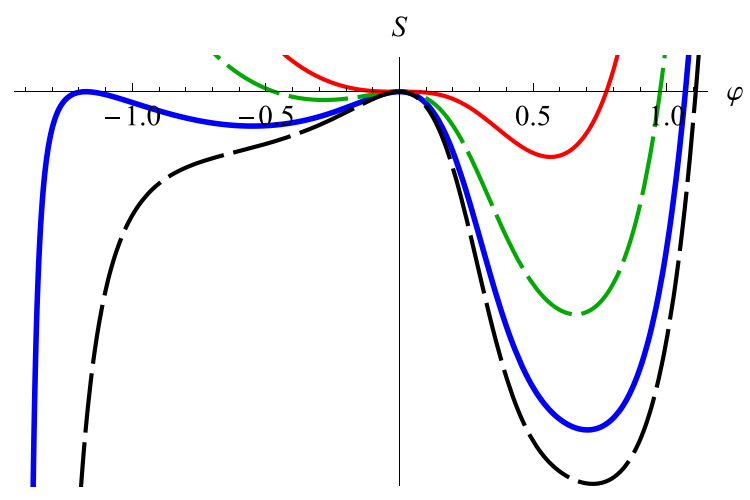

FIG. 7. Typical Sagdeev pseudopotentials for $\beta=0.5$ and $\cos \vartheta=0.8$. The color coding is as follows, from the inside to the outside: (1) solid red at the minimum velocity $M=M_{c}=1.131$, having a triple root at $\varphi=0$, a nonKdV-like positive soliton (amplitude $\varphi=0.774$ ), and a singularity ( $\left.\varphi_{\ell}=-1.552\right)$; (2) dashed green for $M=1.18$, with a nonKdV-like positive soliton $(\varphi=0.975)$, a negative $\mathrm{KdV}$-like soliton $(\varphi=-0.469)$, and a singularity ( $\left.\varphi_{\ell}=-1.472\right)$; (3) solid blue for $M=M_{d l}=1.214$, with a nonKdVlike positive soliton $(\varphi=1.070)$, a negative double layer $\left(\varphi_{d l}=-1.173\right)$, and a singularity $\left(\varphi_{\ell}=-1.418\right)$; (4) dashed black for $M=1.23$, with only a nonKdV-like positive soliton $(\varphi=1.109)$ and a singularity $\left(\varphi_{\ell}=-1.392\right)$.

curves shown in their Figs. 3 and 5. In each case, one finds a singularity in the vicinity of the double layer. These are at $\left|\psi_{\ell}\right| \simeq 0.836-0.838$ for their parameter values. Thus it follows that, as in our case, their model does not support supersolitons. That is in fact confirmed by the hodograph shown in their Fig. 4, where, because of the singularity, the "supersoliton" curve for $M=1.311$ cannot have the necessary closed form associated with a soliton beyond a double layer ${ }^{29}$ or supersoliton. ${ }^{33}$

Repeating the calculation of their singularity, but for $\sigma=0$, we have again found a singularity in each curve, but these have shifted to $0.816-0.817$, showing only a small quantitative difference from the above results for warm ions. Clearly, we are justified in arguing that the assumption of cold ions includes the key qualitative effects and that the introduction of finite ion temperatures does not affect the physics significantly.

The main results found in Fig. 6 will become clearer when other Sagdeev pseudopotentials are added, for the same $\beta=0.5$ and $\cos \vartheta=0.8$, but now for different $M$, as shown in Fig. 7. To begin at the lowest possible $M=M_{c}=1.131$, we have a Sagdeev pseudopotential at the critical minimum speed, denoted by the solid red curve, which has a triple root at $\varphi=0$ and a nonKdV-like positive soliton with amplitude $\varphi=0.774$. As values of $M<M_{c}$ do not give the unstable maximum for Sagdeev pseudopotentials necessary to have solitary wave solutions, this amplitude is the minimum for all positive solutions. That justifies calling such solitons "nonKdV-like": they cannot be obtained from a weakly nonlinear perturbation theory, which requires zero amplitude at the acoustic speed, $M_{C}$.

Next, as $M$ is increased, one finds Sagdeev pseudopotentials with negative and positive solitons, as illustrated by the dashed green curve for $M=1.18$, with a negative KdV-like soliton with amplitude $\varphi=-0.469$ and a positive nonKdVlike soliton with amplitude $\varphi=0.975$.

The double root solution has already been discussed above, and as we now investigate what happens for $M>M_{d l}$, we note that no negative solitons can be generated. This is shown by the graph of the dashed black Sagdeev pseudopotential for $M=1.23$, with only a nonKdV-like positive root at $\varphi=1.109$. There is a negative root at $\varphi=-1.670$, which lies on a branch beyond the singularity at $\varphi_{\ell}=-1.392$ and is thus of no physical relevance. It has been omitted for graphical clarity in Fig. 7 but is included in the hodograph plot, Fig. 8 .

For the same reason most of the range $S>0$ has been omitted, as solitons cannot be obtained there, only possibly nonlinear periodic modes, but these require a different range of integration constants before arriving at a reliable description. Indeed, several papers addressing periodic solutions integrate the intermediate equations with the equivalent of zero boundary conditions far from the "solitary" structure, but then add an arbitrary constant to $S$, which is unfortunately not self-consistent. ${ }^{2-4,6}$ All the Sagdeev pseudopotentials shown here have a fairly strong positive soliton solution, even for $M>M_{d l}$.

In Fig. 8, we show the "phase portrait" corresponding to the different Sagdeev pseudopotentials of Fig. 7, with the same curve coding. Such graphs are often called hodographs, because in the parlance of the classical mechanical analogue of the Sagdeev pseudopotential analysis they represent the "velocity" (here $d \varphi / d x$ ) in terms of the "position" (here $\varphi$ ). We have extended the figure to larger negative $\varphi$, to show the nonphysical root of the dashed black curve at $\varphi=-1.670$, in gray. This is not to be interpreted as being on the "other" side of a very deep well, because there is a singularity at $\varphi=-1.392$, where the Sagdeev pseudopotential goes to $-\infty$. Thus, clearly, the contour for that $M>M_{d l}$ cannot form a closed curve, as required for a supersoliton.

From (17), one can, for the parameter values used for the Sagdeev pseudopotentials in Figs. 7 and 8, calculate $\varphi(x)$ and hence obtain both negative double root and soliton profiles and positive soliton profiles. These are shown in Figs. 9 and 10 , respectively, with the same coding. We shall return to this in Sec. V.

We now treat another example at smaller $\cos \vartheta$, i.e., at larger obliquity, which illustrates the general trends. The hodograph in Fig. 11 is produced for $\cos \vartheta=0.5$ and is the analogue of Fig. 8. Plots for the negative soliton and double layer profiles can easily be generated but look very similar to

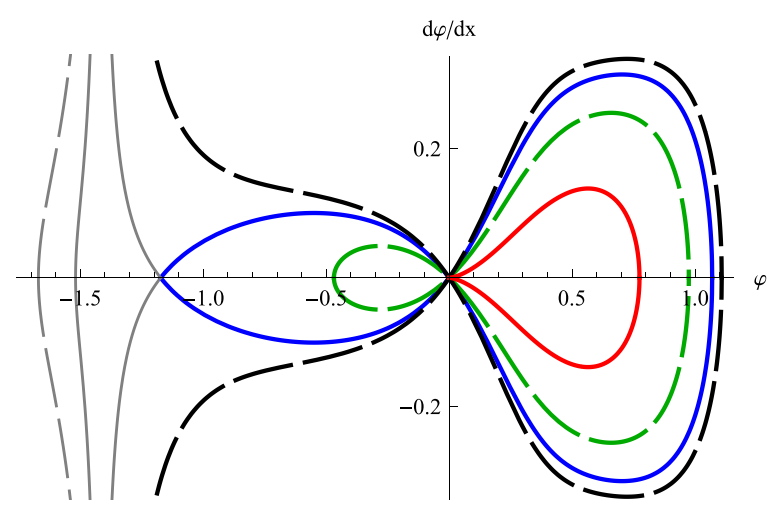

FIG. 8. Hodographs corresponding to the Sagdeev pseudopotentials shown in Fig. 7, for the ranges where those are negative. Nonaccessible $\varphi$ domains are coded in light gray. 


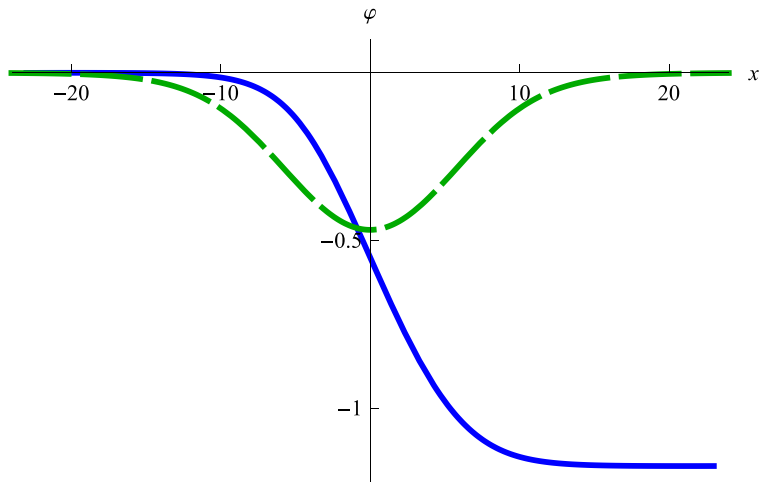

FIG. 9. Double root and soliton profile corresponding to the Sagdeev pseudopotentials having accessible negative roots, as shown in Figs. 7 and 8.

those shown in Fig. 9. A similar remark can be made for the positive nonKdV-like soliton profiles, in comparison to those in Fig. 10. Plots for other values of $\cos \vartheta$ all look qualitatively similar, but are not shown here, to avoid needless repetition.

Over a large range of different Sagdeev pseudopotentials (including numerous examples not shown here), together with the associated hodographs and soliton or double layer profiles, no great qualitative changes are found for varying obliquity. However, it is seen that for increasingly oblique propagation (i.e., $\cos \vartheta$ decreasing), the widths of the double layer profiles increase and the region in potential containing the singularity becomes wider. On the other hand, the double layer amplitudes remain constant, as was also observed in Fig. 2. In contrast to the negative potential structures, the nonKdV-like positive solitons reveal minimal effects due to changes in obliquity. As Fig. 3 also attests, variations in $\beta$ do not show real changes, as long as the obliquity is sufficiently large. We have not been able to find solutions for $S$ at perpendicular propagation, i.e., for $\cos \vartheta=0$.

\section{COMMENTS ON QUASINEUTRALITY}

As a final comment, an apparent dichotomy needs to be mentioned. We have made use of quasineutrality in order to be able to perform the analytical and numerical computations, and that has been in the form of a strict mathematical

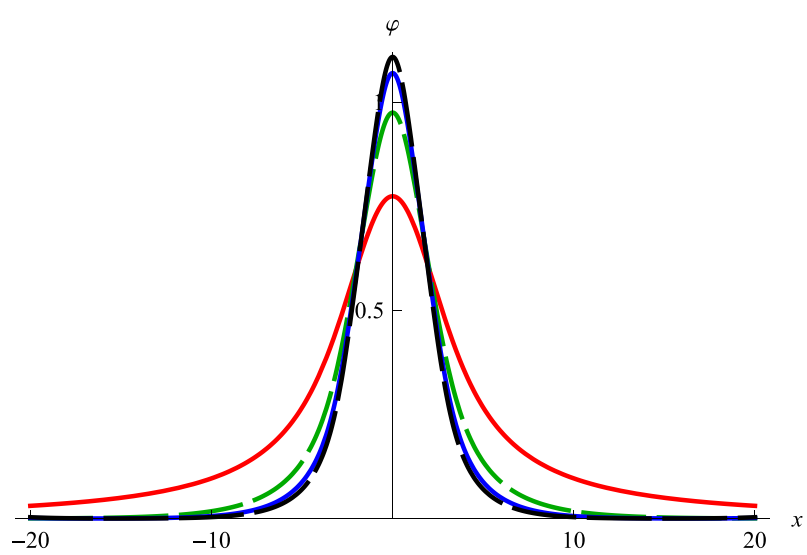

FIG. 10. Positive soliton profiles corresponding to the Sagdeev pseudopotentials shown in Figs. 7 and 8.

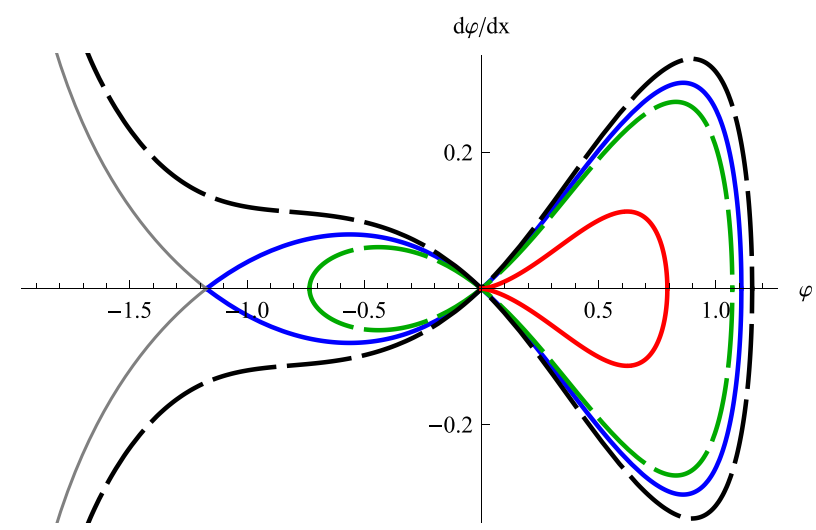

FIG. 11. Typical hodographs for $\beta=0.5$ and $\cos \vartheta=0.5$. The color coding is as follows, from the inside to the outside: (1) solid red at the minimum velocity $M=M_{c}=0.707$, having a triple root at $\varphi=0$, a nonKdV-like positive soliton at $\varphi=0.793$, and a singularity at $\varphi=-2.391$; (2) dashed green for $M=0.75$, with a nonKdV-like positive soliton at $\varphi=1.070$, a negative $\mathrm{KdV}$-like soliton at $\varphi=-0.734$, and a singularity at $\varphi=-2.289$; (3) solid blue for $M=M_{d l}=0.759$, with a nonKdV-like positive soliton at $\varphi=1.109$, a negative double root at $\varphi=-1.173$, and a singularity at $\varphi=-2.269$; (4) dashed black for $M=0.77$, with only a nonKdV-like positive soliton at $\varphi=1.155$ and a singularity at $\varphi=-2.243$.

equality, $n_{i}=n_{e}=n$, in effect rendering the charge density in Poisson's equation (not shown) zero.

Bearing that in mind, one may ask how double layers can occur: after all, the name implies that they are made up of two layers of opposite charge. Furthermore, the curvature observed in soliton profiles, too, represents the effects of a finite local charge density. Yet, we have seen in Sec. IV that physically realistic profiles of both solitons and double layers can indeed be found, starting from (17).

Differentiating (17) with respect to $x$, and assuming that the common factor $d \varphi / d x$ is non-zero, we obtain

$$
\frac{d^{2} \varphi}{d x^{2}}+\frac{d S}{d \varphi}=0
$$

This means that $S^{\prime}$ behaves like an overall charge density, which we had originally neglected by invoking quasineutrality. Equation (27) has been numerically integrated to generate the soliton and double layer profiles shown in Figs. 9 and 10.

However, the analysis is only self-consistent if, in the range of interest for solitons and double layers, $S^{\prime}$ is small enough. To check this, we have plotted in Figs. 12 and 13 this "charge density function" for the Sagdeev pseudopotentials illustrated in Figs. 5 and 7, respectively. For each example, the curves have been drawn from zero to the value(s) of $\varphi$ corresponding to the relevant soliton or double root. This shows that for both the negative soliton and double layer in Fig. $13,\left|S^{\prime}\right|$ is less than or equal to 0.02 , at least in the range of interest. This is probably quite acceptable, as representing an order at which linearization, and even neglect of a term, would be easy to justify. One should note, however, that it does not measure up to typical values of the quasineutrality of stationary plasmas, which may be of order $10^{-6.43}$

On the other hand, for all the positive solitons $\left|S^{\prime}\right|$ shown in the two figures is much larger. In fact, for three of them, it is more than an order of magnitude larger, being about 0.2 to 


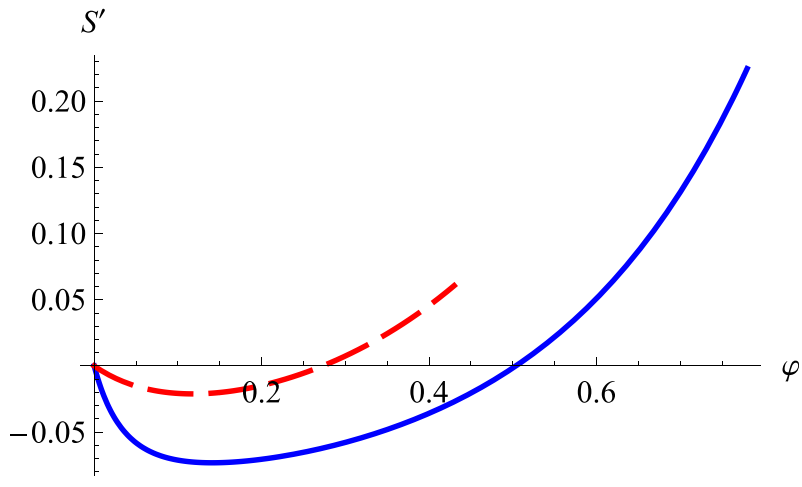

FIG. 12. Derivatives of the Sagdeev pseudopotentials having only positive solitons, as shown in Fig. 5, with corresponding curve coding.

0.4 near the actual roots, that is, at the peak value of the electrostatic disturbance. These large "charge densities" are associated with the deep well in $S$ (i.e., the strong maximum electric field of the soliton, as seen from (17)), and the strong gradient $S^{\prime}$ near the root. How acceptable such large excursions from neutrality are when considering the equivalent charge density to be "small enough" is at best debatable. At worst, they suggest that the approach may be physically inappropriate. Furthermore, the question of how the two domains (positive and negative potential) of the same pseudopotential curve, $S$, with such differing characteristics are to be reconciled, is not obvious.

To the best of our knowledge, such a quantitative a posteriori check on the implications of assuming quasineutrality, when studying solitons propagating obliquely in magnetized plasmas, has not been discussed before.

\section{CONCLUSIONS}

Analyses of the oblique propagation of large amplitude electrostatic waves in a magnetized plasma, as encountered in the literature, use three essential assumptions: that the waves are electrostatic, the plasma response is quasineutral, and the nonlinear structures assume a stationary form in a comoving frame and are thus amenable to a Sagdeev pseudopotential analysis. In view of recent results in the literature, ${ }^{14}$ we have studied the oblique propagation of large amplitude electrostatic waves and solitary structures in a magnetized

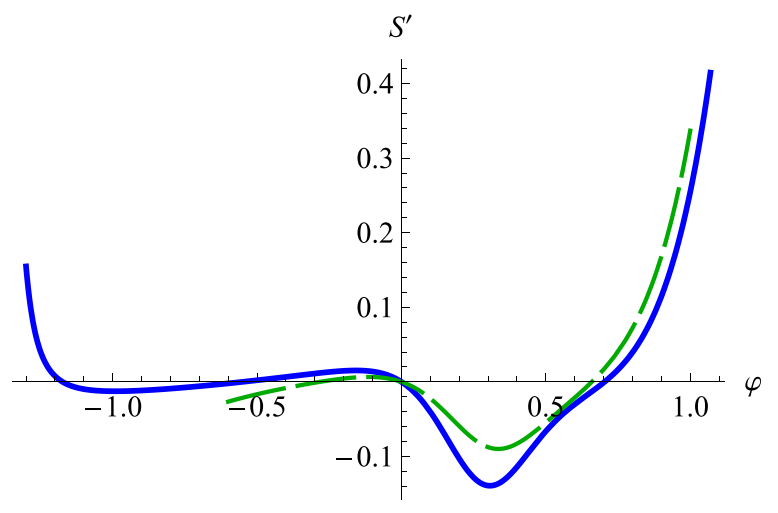

FIG. 13. Derivatives of the Sagdeev pseudopotentials having negative and positive solitons (including possible double layers), as shown in Fig. 7, with corresponding curve coding. plasma, comprising cold fluid ions and Cairns nonthermally distributed electrons. In normalized variables, the resulting Sagdeev pseudopotential $S$ is governed by three essential parameters: the Mach number $M$, the Cairns nonthermal parameter $\beta$, and the angle $\vartheta$ between the directions of propagation and of the static magnetic field, besides the dependent variable, the electrostatic potential $\varphi$.

The standard analysis of the necessary conditions to allow solitary structures yields a minimum $M_{c}$ and a maximum $M_{x}$ for $M$. Between those limits, the Sagdeev pseudopotential has an unstable maximum at the origin, i.e., a double root at $\varphi=0$. However, due to the specific form of the Sagdeev pseudopotential, singularities occur where $S$ becomes infinite (positive or negative) for finite values of $\varphi$. In fact, it has been shown that such singularities will occur not only for Cairns electrons but also for any assumed form of the density $n(\varphi)$ for the inertialess electrons. It is clear that these have a strong influence on the existence range of solitons and double layers, because roots of $S$ beyond the singularity are not accessible from the undisturbed conditions at $\varphi=0$ and therefore have no physical meaning or interest.

As is well known from the properties of the Cairns nonthermal distribution, below a critical $\beta$ only positive compressive solitons are possible, and their amplitudes increase with increasing $\beta, M$, and $\vartheta$. For $M$ close to $M_{c}$, all is well, but near the upper limit $M_{x}$ we have found that the singularities prevent the occurrence of solitons, or at least distort them due to numerical instabilities.

Above the critical $\beta$, there is coexistence between negative rarefactive and positive compressive solitons, and the range of negative solitons, at increasing $M$, ends upon encountering a double layer or a singularity. For an obliquity below $11.8^{\circ}$ the singularity is encountered before a double root can occur, whereas above $31.6^{\circ}$ the double layer is always encountered before the singularity. In the intermediate range there is a crossover: for $\beta$ close to but larger than $\beta_{c}$, double roots are encountered before the singularity, whereas for $\beta$ near the upper limit of $4 / 7$, the singularity prevents the double root from existing. There is always a singularity, for all acceptable $\beta$ and $\vartheta$, and if double roots exist, the graphs of $S(\varphi)$ clearly show the descent of $S$ into the abyss. Hence, no further physically accessible roots for $M>M_{d l}$ are possible. Importantly, this thus rules out the possibility of negative polarity supersolitons. Recent claims of finding supersolitons beyond a double layer ${ }^{14}$ seem to be based on a misinterpretation of the nature of the singularity, assuming it to be merely a deep well.

From a comparison between different Sagdeev pseudopotentials, including numerous examples not shown here, together with the associated hodographs and soliton or double layer profiles, it is seen that for increasingly oblique propagation (i.e., as $\cos \vartheta$ decreases), the double layer amplitude remains constant (as seen also from Fig. 2), but the region in potential containing the singularity becomes wider. The width of the double layer profiles increases with the obliquity. On the other hand, for the nonKdV-like positive solitons, the differences are minimal. All in all, there are no great qualitative changes. Variations in $\beta$ will also not show real changes, as long as the obliquity is sufficiently large. 
We have also addressed an apparent dichotomy, in that we have made use of quasineutrality in order to be able to perform the analytical and numerical computations. We did so in the form of a strict mathematical equality, $n_{i}=n_{e}=n$, setting the charge density in Poisson's equation equal to zero. However, from the Sagdeev pseudopotential formalism we know that $S^{\prime}$ functions as an overall charge density, which we had originally neglected by invoking quasineutrality. When checking whether $S^{\prime}$ remains sufficiently small, we find that for the examples we treated in detail, this is sufficiently well adhered to for the negative solitons and double root solutions, but much less so for the positive solitons. In fact, near the soliton peak, the effective "charge density" calculated post hoc is surprising large, such that it cannot really be neglected. This might point to a need to consider only propagation angles that are not too large, but then the interesting negative double layers might disappear, given that the singularity intervenes first, before the double root.

The constraint due to quasineutrality has to be coupled to earlier warnings ${ }^{8}$ that the neglect of wave magnetic fields, implicit in the use of the electrostatic potential gradient to fully characterize the wave electric field, is only admissible for small nonlinearities and small obliquities. This result was based on investigating qualitative aspects of the ion dynamics.

Our neglect of the magnetic part of the Lorentz force in the electron dynamics, due to the assumption of inertialess electrons, appears not to be justifiable for large angles of propagation. However, this restriction on the admissible obliquity is less stringent than those arising from the quasineutrality and electrostatic electric field assumptions.

Taken together, these comments point to a restricted domain of validity of the standard approach used to describe the oblique propagation of electrostatic waves: the results are only reliable for small enough nonlinearities and small enough obliquity. This holds not only for the problem dealt with in this paper, but for all efforts in the literature based on the combined assumptions of the neglect of wave magnetic field effects and of the use of charge quasineutrality.

\section{ACKNOWLEDGMENTS}

We thank the referee for raising an interesting point that has led to an improvement in the paper. M.A.H. thanks the National Research Foundation of South Africa for partial support under Grant No. 68911. Any opinions, findings, and conclusions expressed herein are those of the authors, and the NRF accepts no liability whatsoever in this regard.

\footnotetext{
${ }^{1}$ M. Y. Yu, P. K. Shukla, and S. Bujarbarua, Phys. Fluids 23, 2146 (1980).

${ }^{2}$ L. C. Lee and J. R. Kan, Phys. Fluids 24, 430 (1981).

${ }^{3}$ Yashvir, T. N. Bhatnagar, and S. R. Sharma, Plasma Phys. Controlled Fusion 26, 1303 (1984).

${ }^{4}$ R. V. Reddy, G. S. Lakhina, N. Singh, and R. Bharuthram, Nonlinear Proc. Geophys. 9, 25 (2002).

${ }^{5}$ S. Mahmood, A. Mushtaq, and H. Saleem, New J. Phys. 5, 28 (2003).
}

${ }^{6}$ J. F. McKenzie, J. Plasma Phys. 70, 533 (2004).

${ }^{7}$ C. R. Choi, D.-Y. Lee, and Y. Kim, J. Astron. Space Sci. 23, 209 (2006).

${ }^{8}$ F. Verheest, J. Phys. A: Math. Theor. 42, 285501 (2009).

${ }^{9}$ S. Sultana, I. Kourakis, N. S. Saini, and M. A. Hellberg, Phys. Plasmas 17, 032310 (2010).

${ }^{10}$ M. Akbari-Moghanjoughi, Phys. Plasmas 18, 032103 (2011)

${ }^{11}$ S. Sultana, I. Kourakis, and M. A. Hellberg, Plasma Phys. Controlled Fusion 54, 105016 (2012).

${ }^{12}$ M. Shahmansouri and H. Alinejad, Astrophys. Space Sci. 344, 463 (2013).

${ }^{13}$ M. Shahmansouri and H. Alinejad, Phys. Plasmas 20, 082130 (2013).

${ }^{14}$ O. R. Rufai, R. Bharuthram, S. V. Singh, and G. S. Lakhina, Phys. Plasmas 23, 032309 (2016).

${ }^{15}$ R. Z. Sagdeev, in Reviews of Plasma Physics, edited by M. A. Leontovich (Consultants Bureau, New York, 1966), Vol. 4, p. 23.

${ }^{16}$ V. M. Vasyliunas, J. Geophys. Res. 73, 2839, doi:10.1029/ JA073i009p02839 (1968).

${ }^{17}$ D. Summers and R. M. Thorne, Phys. Fluids B 3, 1835 (1991).

${ }^{18}$ M. A. Hellberg, R. L. Mace, T. K. Baluku, I. Kourakis, and N. S. Saini, Phys. Plasmas 16, 094701 (2009).

${ }^{19}$ V. Pierrard and M. Lazar, Sol. Phys. 267, 153 (2010).

${ }^{20}$ G. Livadiotis and D. J. McComas, Space Sci. Rev. 175, 183 (2013).

${ }^{21}$ C. Tsallis, J. Stat. Phys. 52, 479 (1988).

${ }^{22}$ J. A. S. Lima, R. Silva, Jr., and J. Santos, Phys. Rev. E 61, 3260 (2000).

${ }^{23}$ F. Verheest, J. Plasma Phys. 79, 1031 (2013).

${ }^{24}$ R. A. Cairns, A. A. Mamun, R. Bingham, R. Boström, R. O. Dendy, C. M. C. Nairn, and P. K. Shukla, Geophys. Res. Lett. 22, 2709, doi:10.1029/ 95GL02781 (1995).

${ }^{25}$ B. Buti, J. Plasma Phys. 24, 169 (1980).

${ }^{26}$ K. Nishihara and M. Tajiri, J. Phys. Soc. Jpn. 50, 4047 (1981).

${ }^{27}$ S. Baboolal, R. Bharuthram, and M. A. Hellberg, J. Plasma Phys. 44, 1 (1990).

${ }^{28}$ T. K. Baluku, M. A. Hellberg, I. Kourakis, and N. S. Saini, Phys. Plasmas 17, 053702 (2010).

${ }^{29}$ T. K. Baluku, M. A. Hellberg, and F. Verheest, Europhys. Lett. 91, 15001 (2010).

${ }^{30} \mathrm{~F}$. Verheest, Phys. Plasmas 18, 083701 (2011).

${ }^{31}$ F. Verheest, M. A. Hellberg, and T. K. Baluku, Phys. Plasmas 19, 032305 (2012).

${ }^{32}$ F. Verheest, Phys. Plasmas 16, 013704 (2009).

${ }^{33}$ A. E. Dubinov and D. Yu. Kolotkov, IEEE Trans. Plasma Sci. 40, 1429 (2012).

${ }^{34}$ A. E. Dubinov and D. Yu. Kolotkov, Plasma Phys. Rep. 38, 909 (2012).

${ }^{35}$ A. Das, A. Bandyopadhyay, and K. P. Das, J. Plasma Phys. 78, 149 (2012).

${ }^{36}$ F. Verheest, M. A. Hellberg, and I. Kourakis, Phys. Plasmas 20, 012302 (2013).

${ }^{37}$ F. Verheest, M. A. Hellberg, and I. Kourakis, Phys. Rev. E 87, 043107 (2013).

${ }^{38}$ F. Verheest, M. A. Hellberg, and I. Kourakis, Phys. Plasmas 20, 082309 (2013).

${ }^{39}$ M. A. Hellberg, T. K. Baluku, F. Verheest, and I. Kourakis, J. Plasma Phys. 79, 1039 (2013).

${ }^{40}$ F. Verheest, G. S. Lakhina, and M. A. Hellberg, Phys. Plasmas 21, 062303 (2014).

${ }^{41}$ F. Verheest and M. A. Hellberg, Phys. Plasmas 22, 012301 (2015).

${ }^{42}$ S. V. Singh and G. S. Lakhina, Commun. Nonlinear Sci. Numer. Simul. 23, 274 (2015).

${ }^{43}$ F. F. Chen, Introduction to Plasma Physics and Controlled Fusion, Vol. 1 Plasma Physics, 2nd ed. (Plenum Press, New York, 1983).

${ }^{44}$ D. B. Melrose, Instabilities in Space and Laboratory Plasmas (Cambridge University, 1986), pp. 209-212.

${ }^{45}$ M. A. Hellberg and R. L. Mace, Phys. Plasmas 9, 1495 (2002).

${ }^{46}$ F. Verheest and M. A. Hellberg, Phys. Plasmas 17, 023701 (2010).

${ }^{47}$ F. Verheest, Phys. Plasmas 17, 062302 (2010).

${ }^{48}$ F. Verheest and M. A. Hellberg, Phys. Plasmas 17, 102312 (2010).

${ }^{49}$ T. K. Baluku and M. A. Hellberg, Plasma Phys. Controlled Fusion 53, 095007 (2011).

${ }^{50}$ F. Verheest and S. R. Pillay, Phys. Plasmas 15, 013703 (2008). 